\title{
AICAR ameliorates high-fat diet-associated pathophysiology in mouse and ex vivo models, independent of adiponectin
}

\author{
Emma Börgeson ${ }^{1,2,3} \cdot$ Ville Wallenius $^{4}$ - Gulam H. Syed ${ }^{5}$ - Manjula Darshi ${ }^{2}$. \\ Juan Lantero Rodriguez ${ }^{1}$ • Christina Biörserud ${ }^{4}$ • Malin Ragnmark Ek ${ }^{4}$. \\ Per Björklund ${ }^{4}$ • Marianne Quiding-Järbrink ${ }^{6}$ - Lars Fändriks ${ }^{4} \cdot$ Catherine Godson $^{7}$ • \\ Kumar Sharma ${ }^{2,3}$
}

Received: 29 August 2016/Accepted: 5 December 2016/Published online: 10 February 2017

(C) The Author(s) 2017. This article is published with open access at Springerlink.com

\begin{abstract}
Aims/hypothesis In this study, we aimed to evaluate the therapeutic potential of 5-aminoimidazole-4-carboxamide ribonucleotide (AICAR), an activator of AMP-activated protein kinase, for ameliorating high-fat diet (HFD)-induced pathophysiology in mice. We also aimed to determine whether the beneficial effects of AICAR were dependent on adiponectin. Furthermore, human adipose tissue was used to examine the effect of AICAR ex vivo.

Methods Six-week-old male C57BL/6J wild-type and Adipo $^{-1-}$ mice were fed a standard-fat diet (10\% fat) or an HFD (60\% fat) for 12 weeks and given vehicle or AICAR $(500 \mu \mathrm{g} / \mathrm{g})$ three times/week from weeks 4-12. Diet-induced pathophysiology was examined in mice after 11 weeks by IPGTT and after 12 weeks by flow cytometry and western blotting. Human adipose tissue biopsies from obese (BMI $35-50 \mathrm{~kg} / \mathrm{m}^{2}$ ) individuals were incubated with vehicle or
\end{abstract}

Catherine Godson and Kumar Sharma are joint senior authors.

Electronic supplementary material The online version of this article (doi:10.1007/s00125-017-4211-9) contains peer-reviewed but unedited supplementary material, which is available to authorised users.

Emma Börgeson

borgesonlab@gmail.com

$\triangle$ Kumar Sharma

kumarsharma@ucsd.edu

1 The Wallenberg Laboratory for Cardiovascular and Metabolic Research, Institute of Medicine, Sahlgrenska Academy, University of Gothenburg, Bruna Stråket 16, S-413 45 Gothenburg, Sweden

2 Centre for Renal Translational Medicine, Institute of Metabolomic Medicine, UC San Diego Health Sciences, San Diego VA HealthCare System, Stein Clinical Research Building, Room 406, mail code 0711, 9500 Gilman Drive, La Jolla, CA 92093, USA
$\operatorname{AICAR}(1 \mathrm{mmol} / \mathrm{l})$ for $6 \mathrm{~h}$ at $37^{\circ} \mathrm{C}$, after which inflammation was characterised by ELISA (TNF- $\alpha$ ) and flow cytometry. Results AICAR attenuated adipose inflammation in mice fed an HFD, promoting an M1-to-M2 macrophage phenotype switch, while reducing infiltration of $\mathrm{CD} 8^{+} \mathrm{T}$ cells. AICAR treatment of mice fed an HFD partially restored glucose tolerance and attenuated hepatic steatosis and kidney disease, as evidenced by reduced albuminuria $(p<0.05)$, urinary $\mathrm{H}_{2} \mathrm{O}_{2}$ $(p<0.05)$ and renal superoxide levels $(p<0.01)$ in both wild-type and Adipo ${ }^{-/-}$mice. AICAR-mediated protection occurred independently of adiponectin, as similar protection was observed in wild-type and Adipoq ${ }^{-/}$mice. In addition, AICAR promoted an M1-to-M2 macrophage phenotype switch and reduced TNF- $\alpha$ production in tissue explants from obese human patients.

Conclusions/interpretation AICAR may promote metabolic health and protect against obesity-induced systemic diseases

3 Veteran's Affairs (VA), San Diego VA HealthCare System, Veterans Medical Research Foundation, San Diego, CA, USA

4 Department of Gastrosurgical Research and Education, Institute of Clinical Sciences, Sahlgrenska Academy, University of Gothenburg, Gothenburg, Sweden

5 Division of Infectious Diseases, School of Medicine, University of California, San Diego, CA, USA

6 Department of Microbiology and Immunology, Institute of Biomedicine, Sahlgrenska Academy, University of Gothenburg, Gothenburg, Sweden

7 University College Dublin (UCD) Diabetes Complications Research Centre, UCD Conway Institute, School of Medicine and Medical Sciences, University College Dublin, Dublin, Ireland 
in an adiponectin-independent manner. Furthermore, AICAR reduced inflammation in human adipose tissue explants, suggesting by proof-of-principle that the drug may reduce obesity-induced complications in humans.

Trial registration: ClinicalTrials.gov NCT02322073

Keywords Adiponectin · AICAR · Inflammation · Kidney disease $\cdot$ Liver disease $\cdot$ Macrophages $\cdot$ Obesity

$\begin{array}{ll}\text { Abbreviations } \\ \text { AICAR } & \text { 5-Aminoimidazole-4-carboxamide ribonucleotide } \\ \text { AMPK } & \text { AMP-dependent protein kinase } \\ \text { CKD } & \text { Chronic kidney disease } \\ \text { DHE } & \text { Dihydroethidium } \\ \text { HFD } & \text { High-fat diet } \\ \text { SFD } & \text { Standard-fat diet } \\ \text { WAT } & \text { White adipose tissue }\end{array}$

\section{Introduction}

The obesity pandemic poses a serious public health challenge. Obesity is associated with numerous pathologies, including diabetes, non-alcoholic fatty liver disease and kidney disease $[1,2]$. There are currently more overweight than underweight people worldwide [3]; this increase in prevalence has prompted a search for effective treatments to tackle obesityrelated pathophysiology.

The adenosine monophosphate analogue 5-aminoimidazole4-carboxamide ribonucleotide (AICAR) activates AMPdependent protein kinase (AMPK), which is a key regulator of energy metabolism and thus a potential target for treatment of obesity-related complications [4, 5]. Evidence from experimental models has shown that AICAR may attenuate metabolic [6-8], hepatic [9-11] and renal pathophysiology [12-15]. However, the use of AICAR could be compromised as some reports indicate that AICAR increases adiponectin production [13]. Although adiponectin is generally described as a protective adipokine [16], several clinical studies have reported a paradoxical inverse association between circulating adiponectin levels and renal function [17, 18]. Specifically, increased adiponectin levels correlate with diabetic nephropathy $[19,20]$, advanced chronic kidney disease (CKD) [21-23] and increased risk of mortality [24]. As we cannot exclude the compromising role of adiponectin in CKD patient groups, it is critical to assess if AICAR-mediated actions are adiponectin dependent to determine its suitability as a drug to target obesity-related pathophysiology.

The aims of this study were to evaluate the therapeutic potential of AICAR for the promotion of metabolic health and reduction of liver and kidney disease in mice fed a high-fat diet (HFD) and to determine if such protection was dependent on adiponectin. Since white adipose tissue (WAT) inflammation is a key driver of obesity-related pathophysiology [25-29], this was characterised in detail. Finally, to translate our rodent data to human pathophysiology, we also investigated whether AICAR could reduce inflammation in omental WAT tissue explants obtained from obese individuals undergoing gastric bypass surgery.

\section{Methods}

Animal study Six-week-old male wild-type (C57BL/6J) and Adipoq $^{-/}$(B6;129-Adipoq ${ }^{\text {tm1Chan } / J) ~ m i c e ~(c a t a l o g u e ~ n u m b e r s ~}$ 000664 and 008195, respectively; The Jackson Laboratory, Sacramento, CA, USA) were housed in a temperature/ humidity controlled room on a $12 \mathrm{~h}$ light $/ 12 \mathrm{~h}$ dark cycle. Mice were allowed to acclimatise for a minimum of one week prior to commencement of experiments, as described in the electronic supplementary material (ESM) Methods. Mice were fed a sucrose-matched standard-fat diet (SFD; $10 \%$ fat) or an HFD (60\% fat) for 12 weeks $(n=3-17$ per experiment, as indicated in the respective figure legends). During weeks 4-12 of SFD/HFD feeding, vehicle or AICAR (500 $\mu \mathrm{g} / \mathrm{g}$ [13]) was given three times per week via i.p. injections. Mice were randomly assigned in consecutive order to an SFD or HFD, and to vehicle or AICAR treatment. At week 11, we performed a fasted IPGTT [30] and collected urine for $24 \mathrm{~h}$ to assess microalbuminuria and urine $\mathrm{H}_{2} \mathrm{O}_{2}$ levels. At the end of the study, WAT and kidney leucocytes were isolated and characterised by flow cytometry. AMPK activation in WAT was determined by western blot analysis. Liver morphology was visualised by haematoxylin and eosin staining and hepatic free cholesterol and triacylglycerol concentrations were measured using standardised kits (cholesterol: WAKO, Richmond, VA, USA; triacylglycerol: Pointe Scientific, Canton, MI, USA). Plasma creatinine was measured by HPLC. In a subset of mice, dihydroethidium (DHE, $50 \mathrm{mg} / \mathrm{kg}$ ) was administered by i.p. injection $16 \mathrm{~h}$ before the mice were killed to quantify renal superoxide levels by confocal imaging ( $n=4$ per group) [14]. Detailed protocols for liver function analysis, HPLC plasma creatinine analysis and renal superoxide measurements are described in ESM Methods.

J774 experiments Serum-starved J774 macrophages were treated with vehicle or AICAR $(1 \mathrm{mmol} / \mathrm{l})$ for $16 \mathrm{~h}$ [31]. Cellular p-AMPK/AMPK was quantified by western blot analysis. Cells were characterised by flow cytometry as proinflammatory $\mathrm{M} 1$ macrophages $\left(\mathrm{CD} 11 \mathrm{c}^{+}\right)$or antiinflammatory M2 macrophages $\left(\mathrm{CD} 206^{+}\right)$; values from vehicle-treated cells were set at $100 \%$.

Human adipose explant culture Omental WAT explants were obtained from obese (BMI $35-50 \mathrm{~kg} / \mathrm{m}^{2}$ ) non-diabetic 
individuals $(n=4)$ undergoing bariatric surgery. WAT explants were incubated ex vivo $(1 \mathrm{~g}$ tissue per $2 \mathrm{ml}$ DMEM) with vehicle or AICAR $(1 \mathrm{mmol} / \mathrm{l})$ for $6 \mathrm{~h}$ at $37^{\circ} \mathrm{C}$ (see ESM Methods for further details). Supernatant TNF- $\alpha$ levels were determined by ELISA and tissue leucocytes were characterised by flow cytometry.

Leucocyte phenotyping by flow cytometry Tissue leucocytes were isolated by treating WAT and kidneys with collagenase $\left(5 \mathrm{mg} / \mathrm{ml}\right.$ and $10 \mathrm{mg} / \mathrm{ml}$, respectively) at $37^{\circ} \mathrm{C}$ $[29,32]$. Lysates were filtered ( $70 \mu \mathrm{m}$ pore size) and $5 \times 10^{5}$ cells were stained by Aqua-Live-Dead (Thermo Fisher, Waltham, MA, USA) and relevant antibodies for characterisation (see ESM Table 1 and ESM Table 2 for antibody details). Both human and murine lymphocytes $\left(\mathrm{CD}^{+} \mathrm{CD} 45^{+}\right)$ were characterised as $\mathrm{T}$ helper $\left(\% \mathrm{CD} 4^{+}\right.$of $\left.\mathrm{CD}^{+} \mathrm{CD} 45^{+}\right)$ and cytotoxic $\mathrm{T}$ cells $\left(\% \mathrm{CD} 8^{+}\right.$of $\left.\mathrm{CD}^{+} \mathrm{CD}^{+} 5^{+}\right)$. In murine tissues, $\mathrm{F} 4 / 80^{+}$macrophages $\left(\mathrm{CD} 45^{+} \mathrm{F} 480^{+}\right)$were subcategorised as inflammatory M1 macrophages (\% $\mathrm{CD} 11 \mathrm{c}^{+}$of $\mathrm{CD} 45^{+} \mathrm{F} 480^{+}$) or $\mathrm{M} 2$ macrophages $\left(\% \mathrm{CD} 206^{+}\right.$ of $\mathrm{CD} 45^{+} \mathrm{F} 480^{+}$). In human WAT, the mononuclear/ macrophage population $\left(\mathrm{CD} 45^{+}\right)$was identified as M1 macrophages $\left(\% \mathrm{CD} 11 \mathrm{c}^{+}\right.$of $\left.\mathrm{CD} 45^{+}\right), \mathrm{M} 2 \mathrm{a}$ macrophages $(\%$ $\mathrm{CD} 206^{+}$of $\left.\mathrm{CD} 45^{+}\right), \mathrm{M} 1 / \mathrm{M} 2 \mathrm{~b}$ macrophages $\left(\% \mathrm{CD} 86^{+}\right.$of $\left.\mathrm{CD} 45^{+}\right)$or $\mathrm{M} 2 \mathrm{a} / \mathrm{M} 2 \mathrm{c}$ macrophages $\left(\% \mathrm{CD} 163^{+}\right.$of $\left.\mathrm{CD} 45^{+}\right)$. Gating was determined using Fluorescence-Minus-One controls (see ESM Methods for further details).

Western blot analysis Adipose tissue and serum-starved J774 macrophages were homogenised in RIPA lysis buffer. Briefly, $40 \mu \mathrm{g}$ protein was loaded onto a $16 \%$ SDS-PAGE gel and transferred onto polyvinylidene difluoride (PVDF) membranes $(0.2 \mu \mathrm{m}$ pore size) [31]. Proteins were identified using rabbit anti-p-AMPK $\alpha$ (Thr172; no. 2535s), rabbit anti-AMPK $\alpha$ (no. 2532s) and rabbit anti-adiponectin (no. 2789) antibodies (all diluted 1:1000; Cell Signaling, Danvers, MA, USA). Proteins were normalised against $\beta$-actin (mouse anti- $\beta$-actin antibody [no. A2228]; Sigma, St Louis, MO, USA), diluted 1:10,000 (see ESM Methods for further details). Original western blot images were cropped as indicated by vertical lines.

Study approval The Veterans Affairs San Diego Healthcare System Institutional Animal Care and Use Committee (IACUC) approved all animal procedures (approval no. 10-029), and the Guide for the Care and Use of Laboratory Animals was followed during experiments. Human tissue specimens were obtained from a larger study (ClinicalTrials.gov NCT02322073) in agreement with the principal investigator, V. Wallenius. The Regional Ethical Review Board (Gothenburg, Sweden) approved all study procedures (Dnr 682-14) and all patients were enrolled in accordance with the Helsinki Declaration. Written informed consent was obtained from all participants included in this study.
Statistical analyses Gaussian distribution was assumed and two-tailed Student's $t$ test, or two-way ANOVA with paired Bonferroni correction as a post hoc comparison was used, as indicated in the figure legends. Analyses were performed using GraphPad Prism version 5 (La Jolla, CA, USA), licensed to UC San Diego.

\section{Results}

AICAR attenuated HFD-induced adipose inflammation independent of adiponectin Wild-type and Adipo ${ }^{-/-}$mice were fed a sucrose-matched SFD (10\% fat) or HFD (60\% fat) for 12 weeks (ESM Fig. 1a). Because this HFD regime has previously been shown to cause systemic disease after 4 weeks, such as renal impairment [13], we initiated AICAR treatment at week 4 to test the effect of AICAR as an intervention.

As expected, HFD-fed mice gained significantly more weight than mice fed an SFD (ESM Fig. 1b). AICAR is known to increase metabolism and weight loss [7], even in sedentary mice [8]. Accordingly, we observed that AICAR attenuated weight gain during the final few weeks of the diet regimen, both in wild-type and Adipoq ${ }^{-/-}$mice (ESM Fig. 1b). However, AICAR-treated HFD-fed mice weighed significantly more than controls fed an SFD in both mouse strains throughout the study (ESM Fig.1b).

The total number of $\mathrm{F} 4 / 80^{+}$macrophages was not affected by HFD in perigonadal WAT from wild-type mice (Fig. 1a), in accordance with our previous studies [29]. However, HFD-fed Adipo $q^{-1-}$ mice presented with an increased number of $\mathrm{F} 4 / 80^{+}$ macrophages $\left(p<0.05\right.$; Fig. 1a). Adipo $q^{-1-}$ mice fed either diet exhibited a higher percentage of $\mathrm{CD} 11 \mathrm{c}^{+} \mathrm{M} 1$ macrophages compared with their respective wild-type controls $(p<0.001$; Fig. 1b).

AICAR attenuated HFD-induced WAT inflammation in both mouse strains; in wild-type mice, AICAR treatment reduced the percentage of $\mathrm{CD} 11 \mathrm{c}^{+} \mathrm{M} 1$ macrophages $(p<0.01)$ and increased levels of anti-inflammatory CD206 ${ }^{+}$ M2 macrophages $(p<0.001$; Fig. $1 b-d)$. Similarly, AICAR attenuated HFD-induced CD11 $\mathrm{c}^{+}$M1 macrophages in Adipo $^{-1-}$ mice $(p<0.001)$. Macrophage $\mathrm{CD}^{206^{+}}$expression was increased in SFD-fed Adipoq ${ }^{-/}$mice compared with SFD-fed wild-type mice, but there were no changes between Adipoq $^{-/-}$mice on an SFD or an HFD with or without AICAR treatment (Fig. 1c). AICAR also reduced the percentage of cytotoxic $\mathrm{CD}^{+} \mathrm{T}$ cells in both mouse strains but did not affect levels of $\mathrm{CD}^{+} \mathrm{T}$ cells (Fig. 1e-h). Although we and others have previously shown that HFD reduces p-AMPK/AMPK [13], surprisingly HFD did not alter AMPK activity in WAT in the present study. This may be explained by the fact that we matched sucrose levels in SFD and HFD regimens in the present study. However, as expected, AICAR increased WAT 


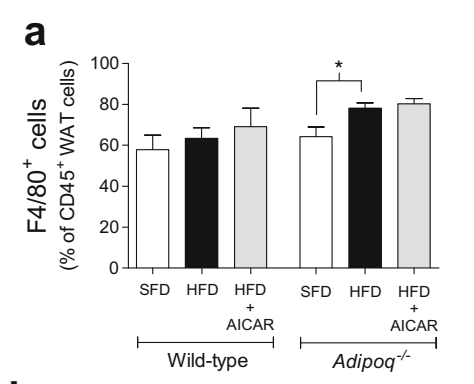

d

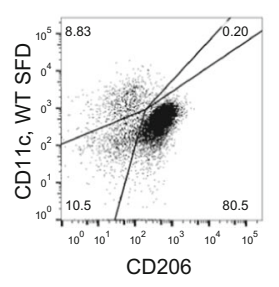

e

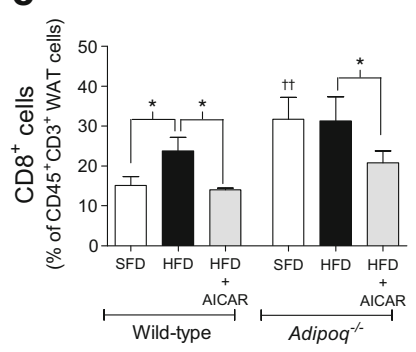

b

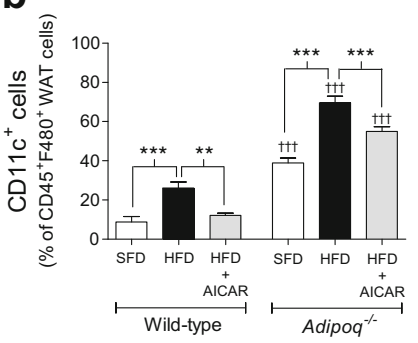

C

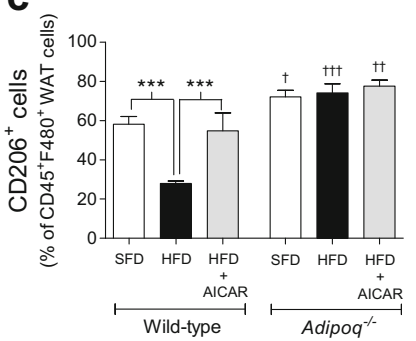

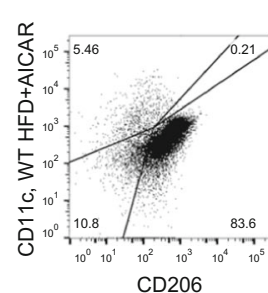

f

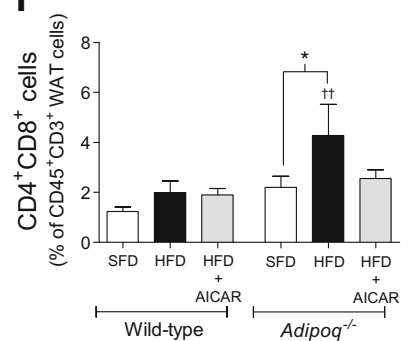

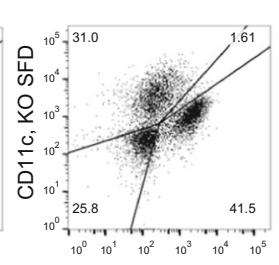

CD206

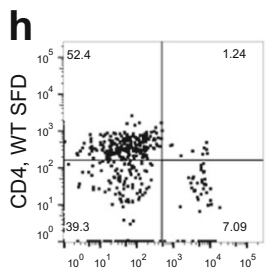

CD8

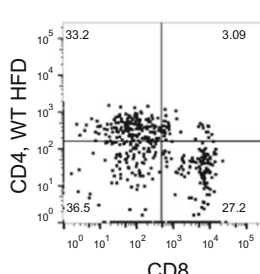

CD8

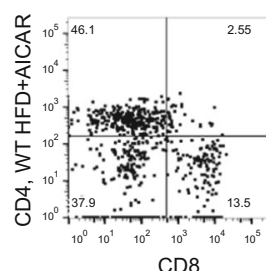

CD8

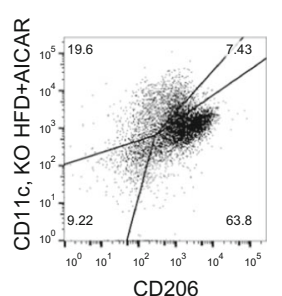

CD206
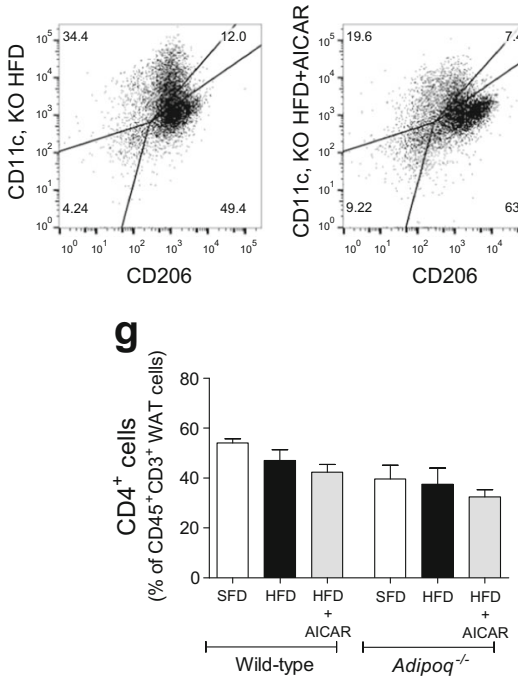

i
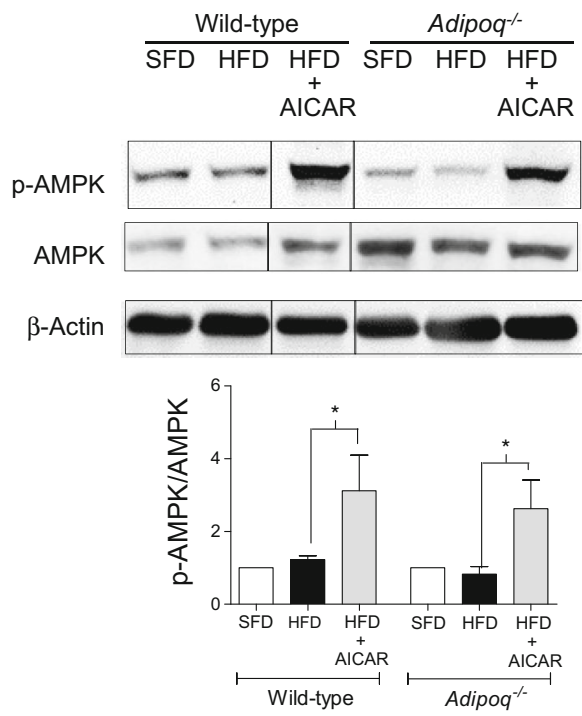

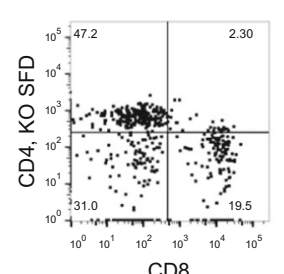

CD8

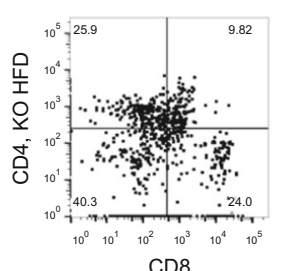

CD8

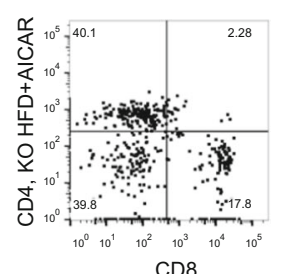

CD8 j
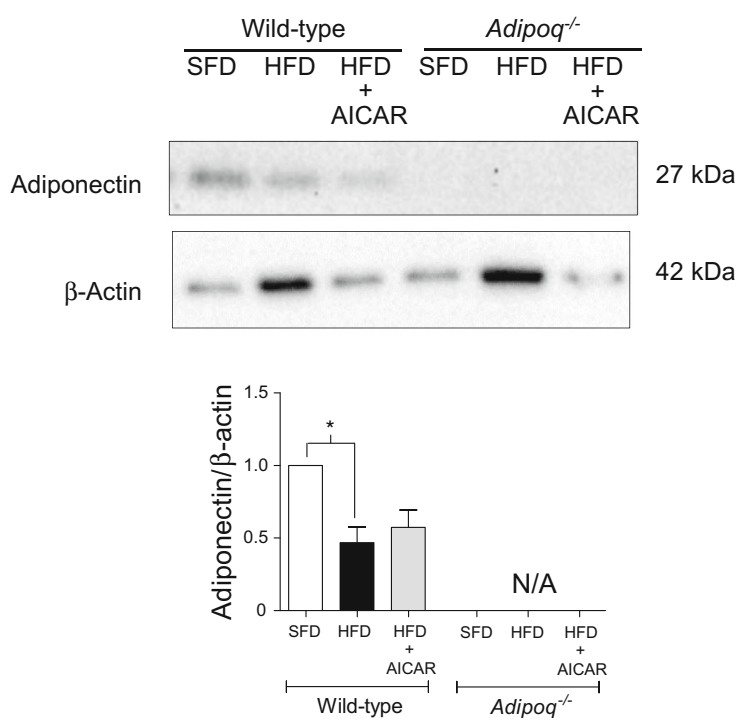
Fig. 1 AICAR attenuates adipose inflammation independent of adiponectin in obese mice. Wild-type and Adipo ${ }^{-1-}$ mice fed a 12 week SFD (10\% fat) or HFD ( $60 \%$ fat) received vehicle or AICAR $(500 \mu \mathrm{g} / \mathrm{g})$ during weeks $4-12$. WAT leucocytes were characterised by flow cytometry; (a)-(d) Macrophages were characterised as pan-macrophages $\left(\mathrm{F} 4 / 80^{+}\right)$, proinflammatory $\mathrm{M} 1$-macrophages $\left(\mathrm{CD} 11 \mathrm{c}^{+}\right)$or anti-inflammatory M2-macrophages $\left(\mathrm{CD} 206^{+}\right)(n=5)$. (e)-(h) Lymphocytes were characterised as $\mathrm{T}$ killer $\left(\mathrm{CD} 8^{+}\right)$vs $\mathrm{T}$ helper $\left(\mathrm{CD}^{+}\right)$cells $(n=5)$. (i), (j) AMPK activation and adiponectin levels were analysed by western blot. AMPK blots were cut as indicated (full blots and details of cutting are presented in ESM Fig. 4) $(n=3)$. Data are presented as mean \pm SEM. $* p<0.05, * * p<0.01, * * * p<0.001$; ANOVA with Bonferroni correction; ${ }^{\dagger} p<0.05,{ }^{\dagger \dagger} p<0.01,{ }^{\dagger \dagger} p<0.001$, Adipo $^{-1-}$ vs wild-type mice for respective conditions. N/A, not applicable

p-AMPK/AMPK levels (Fig. 1i). Furthermore, AICAR did not restore HFD-mediated attenuation of WAT adiponectin in wild-type mice (Fig. 1j).

WAT comprises a myriad of cells, including adipocytes, epithelial cells and leucocytes. To determine if AICAR could alter the macrophage phenotype via direct or indirect effects, we also investigated AICAR-mediated effects on murine macrophages in vitro, using the $\mathrm{J} 774$ cell line. Similar to the in vivo findings, AICAR promoted an M1-to-M2 phenotype switch in cultured macrophages, attenuating $\mathrm{CD} 11 \mathrm{c}^{++}$expression $(p<0.01)$, while promoting CD206 ${ }^{+}$expression $(p<0.05$; Fig. 2a,b). This correlated with increased p-AMPK/AMPK in this cell line $(p<0.05$; Fig. $2 c, d)$, but AMPK/ $\beta$-actin remained unaltered (Fig. 2c,e).

AICAR partially restored glucose tolerance in obese mice independent of adiponectin An IPGTT was performed to assess glucose tolerance (Fig. 3a-c). HFD significantly impaired glucose clearance in both wild-type and Adipoq ${ }^{-/-}$ mice $(p<0.001)$. However, HFD-fed Adipo $q^{-/-}$mice presented with exaggerated glucose intolerance compared with HFD wild-type controls $(p<0.05)$ (Fig. 3c).

AICAR did not significantly alter the HFD-induced increase in fasting blood glucose in either mouse strain (Fig. 3a,b). However, AICAR partially restored HFDinduced impairment of glucose clearance in wild-type mice $(p<0.05)$. This AICAR-mediated beneficial effect on glucose clearance was sustained in the Adipo $q^{-/}$mice $(p<0.05$; Fig. 3c) and significantly lower levels of blood glucose were observed in AICAR-treated vs untreated HFD Adipo ${ }^{-1-}$ at $60 \mathrm{~min}$ and $120 \mathrm{~min}$ post-glucose injection (Fig. 3b).

AICAR attenuated HFD-induced hepatic steatosis independent of adiponectin AICAR reduced HFD-induced hepatic steatosis, as evidenced by reduced hepatic vacuolisation (Fig. 4a) and triacylglycerol content (Fig. 4b). The drug also attenuated HFD-induced elevations in hepatic cholesterol levels in Adipoq ${ }^{-/-}$mice $(p<0.05$; Fig. 4c). Furthermore, AICAR-mediated attenuation of hepatic vacuolisation was more pronounced in Adipoq ${ }^{-1-}$ mice (Fig. 4a). Liver weight/hypertrophy was not altered by AICAR treatment (ESM Fig. 2).

AICAR attenuated HFD-induced kidney disease independent of adiponectin Wild-type and Adipoq ${ }^{-/-}$mice developed significant renal dysfunction following a 3 month HFD regimen, as evidenced by increased albuminuria, urine $\mathrm{H}_{2} \mathrm{O}_{2}$ and renal superoxide production compared with SFD (Fig. 5a,b,d), without changes in plasma creatinine (ESM Fig. 3). Furthermore, renal hypertrophy was increased in HFD-fed Adipoq ${ }^{-/}$mice compared with SFD (Fig. 5c). The total number of renal $\mathrm{F} 4 / 80^{+}$pan-macrophages was not altered by HFD in either mouse strain (Fig. 5e), but HFD significantly
Fig. 2 AICAR promotes AMPK activation and an M1-to-M2 phenotype switch in cultured macrophages. J774 macrophages were incubated with vehicle or $\operatorname{AICAR}(1 \mathrm{mmol} / \mathrm{l})$ for $16 \mathrm{~h}$ $(n=3)$. Cells were characterised as (a) proinflammatory M1 $\left(\mathrm{CD} 11 \mathrm{c}^{++}\right)$or $(\mathbf{b})$ antiinflammatory M2 $\left(\mathrm{CD} 206^{+}\right)$by flow cytometry and $(\mathbf{c}-\mathbf{e})$ p-AMPK/AMPK was assessed by western blot. Data are presented as mean \pm SEM. $* p<0.05$, $* * p<0.01$, paired Student's $t$ test
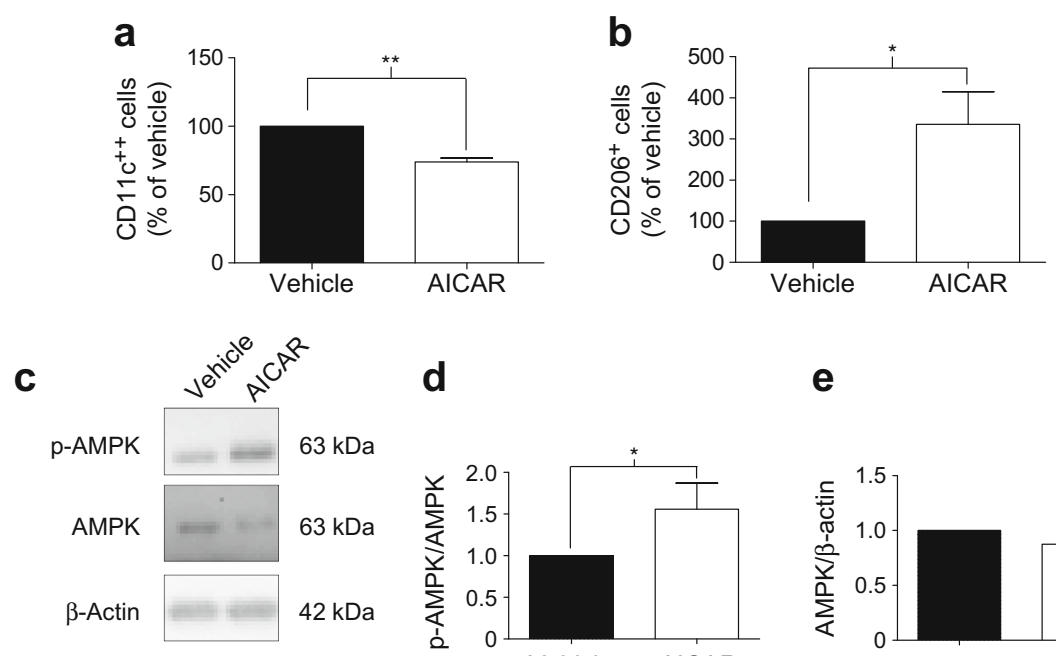

d

e
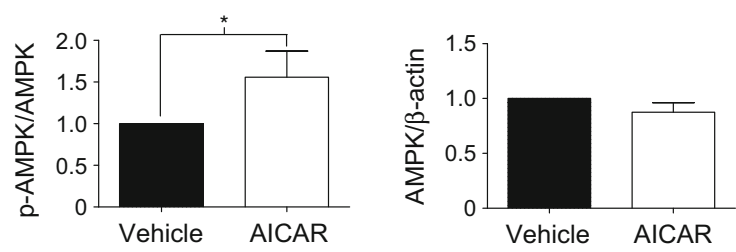
a

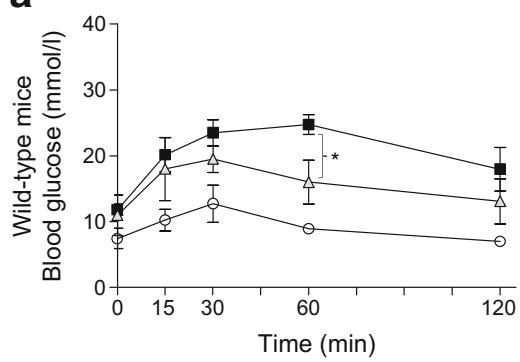

b

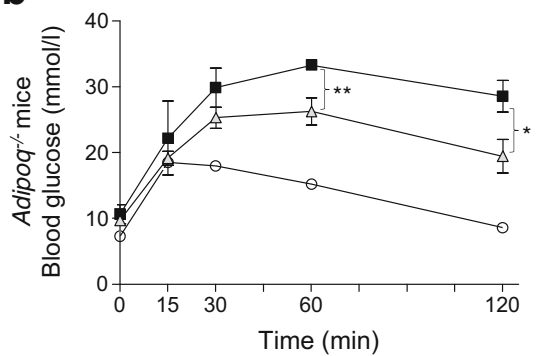

C

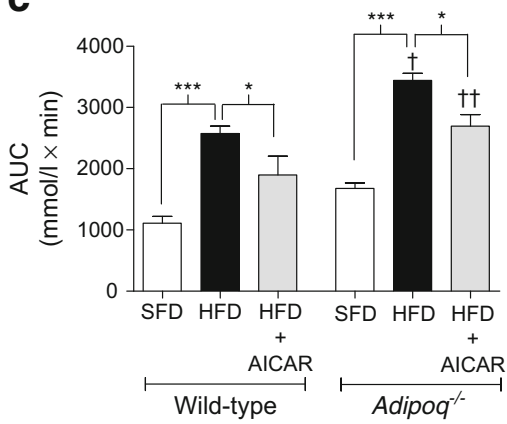

Fig. 3 AICAR partially restores glucose tolerance independent of adiponectin in obese mice. Wild-type and Adipoq ${ }^{-1-}$ mice fed a 12 week SFD (10\% fat) or HFD (60\% fat) received vehicle or AICAR $(500 \mu \mathrm{g} / \mathrm{g})$ during weeks 4-12 of feeding. Glucose tolerance was tested at week 11 by an IPGTT in (a) wild-type ( $n=4$ for all groups) and (b) Adipoq ${ }^{-/-}$

increased renal CD11 $\mathrm{c}^{+} \mathrm{M} 1$ macrophages in wild-type mice (Fig. 5f,g). Renal CD11 $\mathrm{c}^{+}$M1 macrophage levels were higher in Adipo ${ }^{-/-}$mice compared with wild-type mice fed an SFD, but no additional increase was observed in HFD-fed Adipo ${ }^{-/}$ mice (Fig. 5f,h).

AICAR significantly attenuated HFD-induced albuminuria (Fig. 5a), urinary $\mathrm{H}_{2} \mathrm{O}_{2}$ (Fig. 5b) and renal superoxide (Fig. 5d) in both wild-type and Adipoq ${ }^{-1-}$ mice. Furthermore, AICAR attenuated HFD-induced renal hypertrophy in Adipoq $^{-1-}$ mice (Fig. 5c). Finally, AICAR completely attenuated the HFD-induced increase in renal CD11 ${ }^{+}$M1 macrophages in the wild-type strain (Fig. 5f).
( $n=3$ for SFD and HFD, $n=7$ for HFD + AICAR) mice. Circles, SFD; squares, HFD; triangles, HFD + AICAR. (c) Graph of the AUC of IPGTT curves. Data are presented as mean \pm SEM. $* p<0.05, * * p<0.01$, $* * * p<0.001$, ANOVA with Bonferroni correction; ${ }^{\dagger} p<0.05,{ }^{\dagger \dagger} p<0.01$, Adipo $^{-/-}$vs wild-type mice for respective conditions

AICAR reduced adipose inflammation in tissue explants obtained from obese human patients WAT inflammation is a key driver of obesity-related pathophysiology [26-29]. As a proof-of-principle for translating our rodent data to human pathophysiology, we investigated whether AICAR could reduce inflammation and manipulate leucocyte phenotypes in omental WAT explants taken from obese patients undergoing gastric bypass surgery.

The antigens used to phenotype human macrophages varied slightly from the panel used for mice. Thus, we characterised human macrophages using the following activation markers: $\mathrm{CD} 11 \mathrm{c}^{+}(\mathrm{M} 1), \mathrm{CD}^{+} 6^{+}(\mathrm{M} 1 / \mathrm{M} 2 \mathrm{~b})$,
Fig. 4 AICAR attenuates hepatic steatosis independent of adiponectin in obese mice. Wildtype and Adipoq ${ }^{-1-}$ mice fed a 12 week SFD (10\% fat) or HFD (60\% fat) received vehicle or AICAR $(500 \mu \mathrm{g} / \mathrm{g})$ during weeks 4-12. (a) Representative images of hepatic haematoxylin and eosin staining (magnification $\times 40$ ). Hepatic (b) triacylglycerol and (c) cholesterol $(n=3)$. Data are presented as mean \pm SEM. $* p<0.05, * * p<0.01$, ANOVA with Bonferroni correction
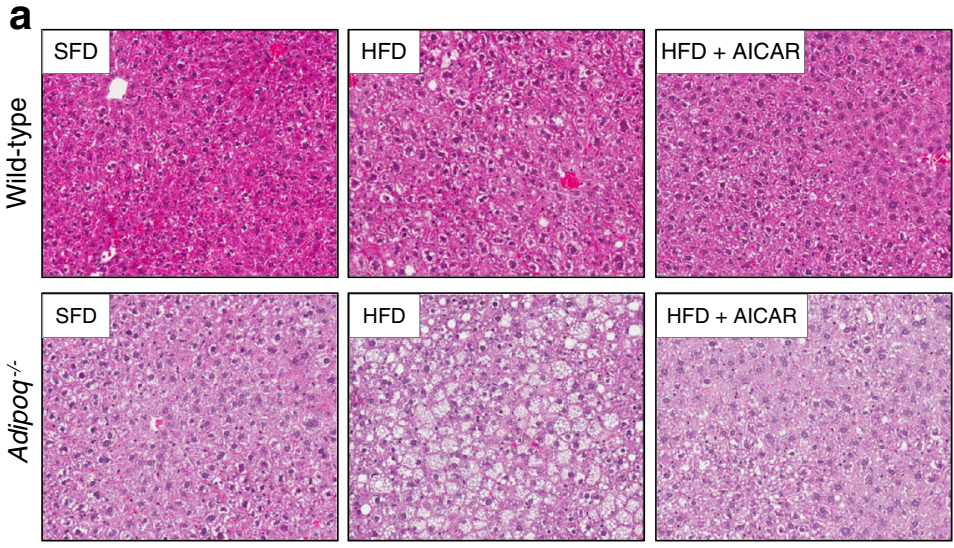

b

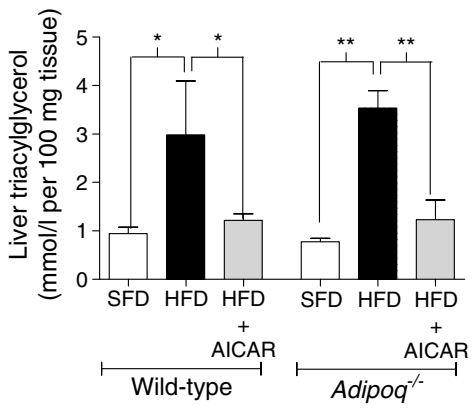

C

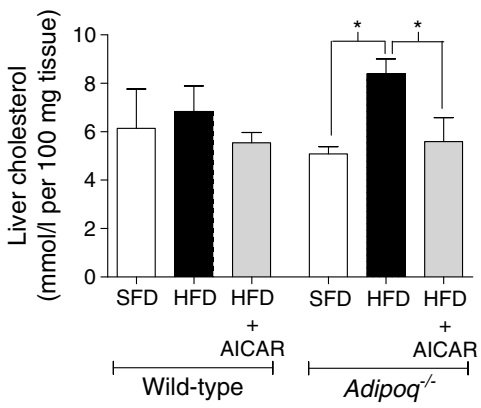



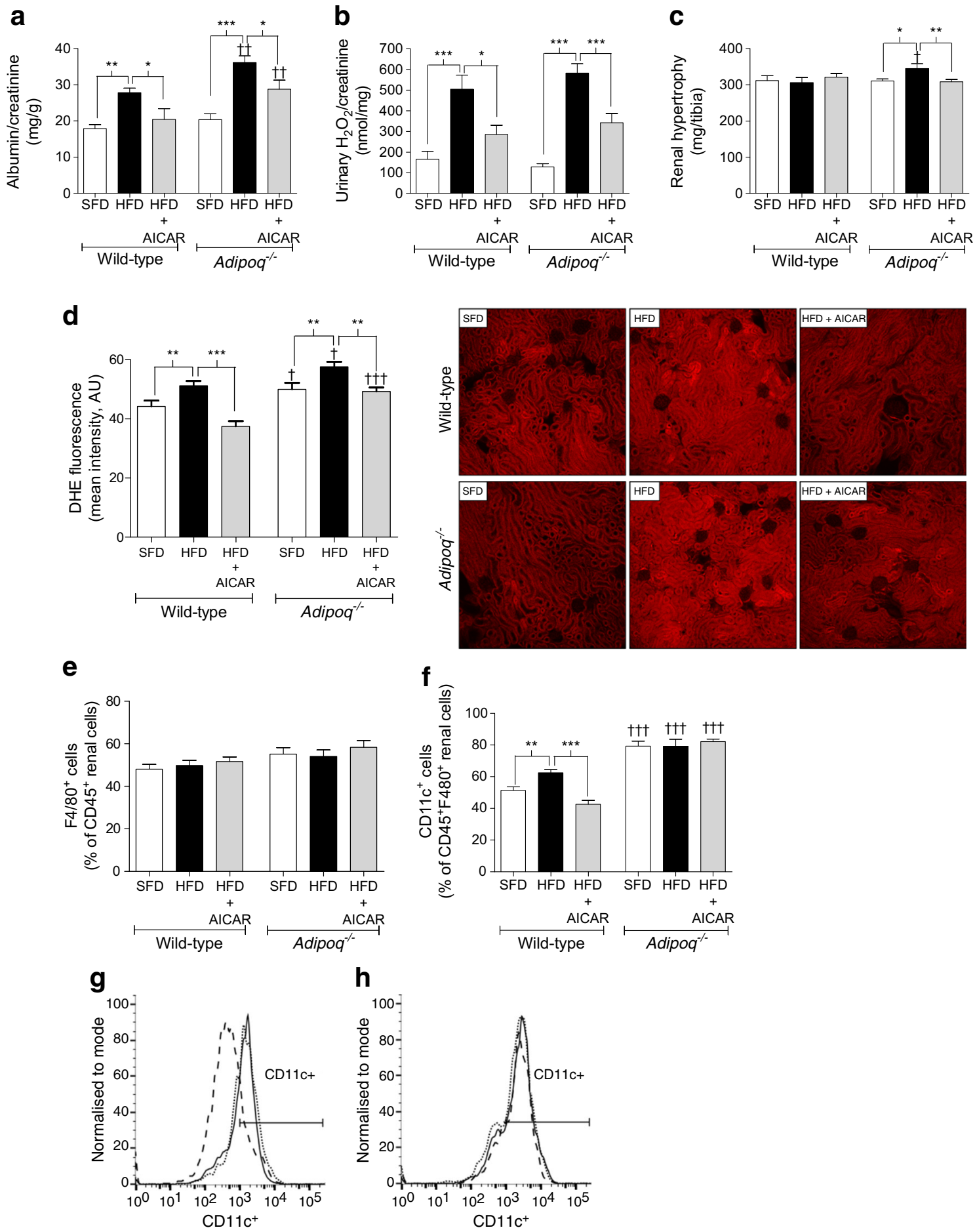

Fig. 5 AICAR attenuates kidney disease independent of adiponectin in obese mice. Wild-type and Adipoq ${ }^{-1-}$ mice fed a 12 week SFD (10\% fat) or HFD (60\% fat) received vehicle or AICAR $(500 \mu \mathrm{g} / \mathrm{g})$ during weeks 4-12. (a) Micro-albuminuria, (b) urine $\mathrm{H}_{2} \mathrm{O}_{2}$ and (c) renal hypertrophy were assessed $(n=7)$. (d) DHE was injected $16 \mathrm{~h}$ prior to killing the mice and renal DHE oxidation was quantified as a measurement of superoxide production (magnification $\times 100 ; n=4$ ). (e) Renal pan-macrophages $\left(\mathrm{F} 4 / 80^{+}\right)$and (f) proinflammatory M1 macrophages $\left(\mathrm{CD} 11 \mathrm{c}^{+}\right)$were

$\mathrm{CD}^{206}{ }^{+}(\mathrm{M} 2 \mathrm{a})$ or CD163 ${ }^{+}(\mathrm{M} 2 \mathrm{a} / \mathrm{M} 2 \mathrm{c})$. This classification is based on the Martinez et al scheme, whereby M1 characterised and quantified by flow cytometry $(n=5)$. $(\mathbf{g}-\mathbf{h})$ Flow cytometry histogram showing number of $\mathrm{CD} 11 \mathrm{c}^{+}$positive cells in $(\mathbf{g})$ wild-type and (h) Adipoq $^{-1-}$ mice, incubated with vehicle (dotted line), fed an HFD without AICAR (solid line), or fed an HFD with AICAR (dashed line). Data are presented as mean \pm SEM. $* p<0.05, * * p<0.01$, $* * * p<0.001$, ANOVA with Bonferroni correction; ${ }^{\dagger} p<0.05,{ }^{\dagger \dagger} p<0.01$, ${ }^{\dagger \dagger} p<0.001$, Adipo $^{-1-}$ vs wild-type mice for respective conditions

macrophages display potent inflammatory activities, whereas $\mathrm{M} 2 \mathrm{a}$ and M2c macrophages downregulate proinflammatory 
cytokines and promote resolution. Meanwhile, M2b macrophages produce IL-12 and IL-10, and are not antiinflammatory per se, but rather activate the adaptive $\mathrm{B}$ cell responses and regulate $\mathrm{B}$ cell and $\mathrm{T}$ cell trafficking [33].

Similar to our findings in mice, compared to vehicle AICAR promoted a shift towards inflammatory resolution in human WAT by increasing the percentage of antiinflammatory $\mathrm{CD}^{2} 06^{+}$macrophages $(p<0.05)$, while reducing the percentage of proinflammatory $\mathrm{CD} 86^{+}$macrophages $(p<0.05)$. However, AICAR did not affect human CD11 $\mathrm{c}^{+}$or $\mathrm{CD} 163^{+}$macrophage expression (Fig. 6a,b), or the $\mathrm{CD} 8^{+}$or $\mathrm{CD}^{+}{ }^{+} \mathrm{T}$ cell populations (Fig. $6 \mathrm{c}, \mathrm{d}$ ) in this $6 \mathrm{~h}$ ex vivo experiment. However, AICAR did reduce TNF- $\alpha$ secretion compared to vehicle $(p<0.001$; Fig. 6 e) .

\section{Discussion}

Obesity is an independent risk factor for numerous pathologies, including diabetes and liver and kidney disease $[1,2]$. As the prevalence of obesity is increasing worldwide [3], the search for effective treatments against obesity-related pathophysiology is ongoing. Here we demonstrate that the AMPK-activating drug AICAR has therapeutic potential in this context. AICAR attenuates HFD-induced WAT inflammation and pathophysiology associated with diabetes, and liver and kidney disease in an adiponectin-independent manner. Collectively, these findings support a therapeutic potential for AICAR in attenuating HFD-induced pathophysiology (summarised in Fig. 7).

AICAR has previously been reported to increase metabolism and weight loss [7], even in sedentary mice [8]. Thus, it is not surprising that AICAR-treated HFD-fed mice gained less weight during the last weeks of the diet regimen, compared with vehicle-treated HFD-fed control mice. This effect on weight gain may have mediated some of the observed beneficial effects of AICAR, but it is unlikely that this is the sole protective mechanism of this compound since AICAR-treated HFD-fed mice weighed significantly more than controls fed an SFD.

Our observation that AICAR attenuates WAT inflammation may indicate a key mechanism of action as obesity-induced adipose inflammation is known to promote systemic pathophysiology [26, 27, 29, 34]. Indeed, inflammatory M1 a
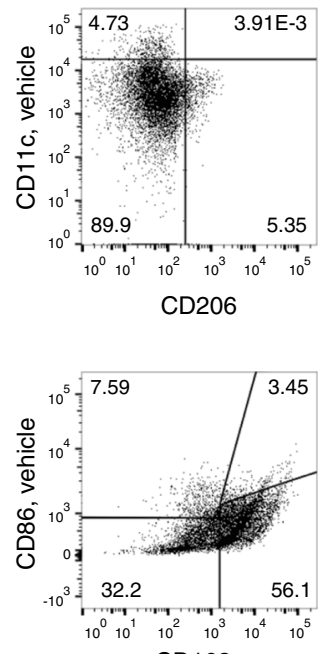

CD163
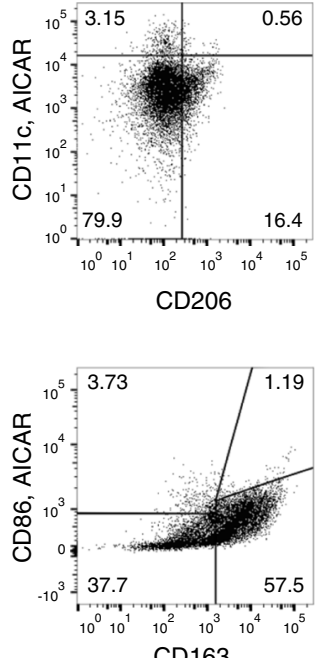

b
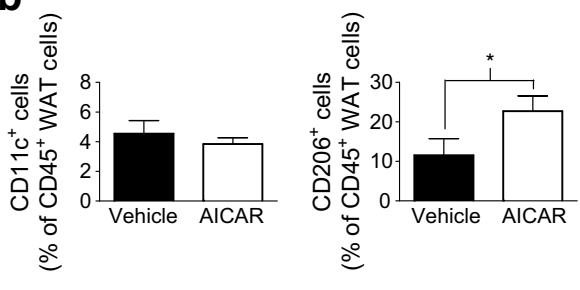
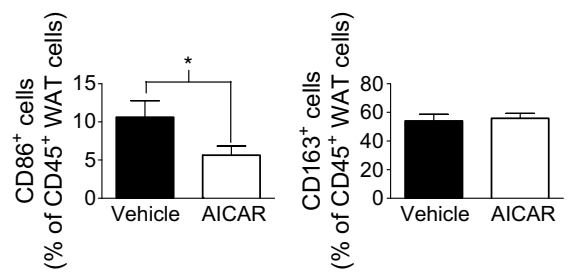

C

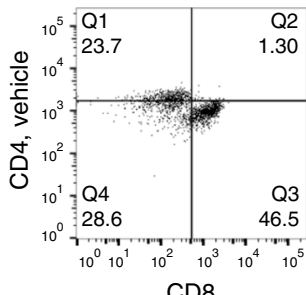

CD8

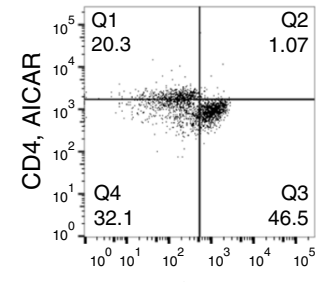

CD8 d

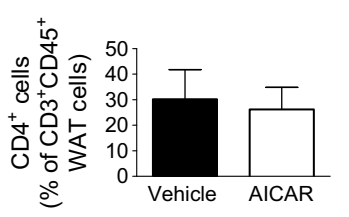

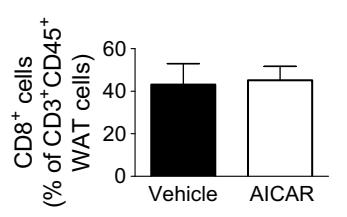

e

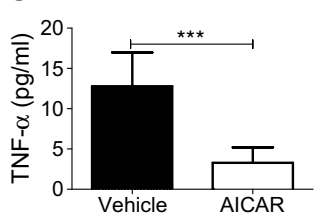

Fig. 6 AICAR reduces inflammation in adipose explants from obese individuals. Omental WAT explants from obese individuals (BMI $35-50 \mathrm{~kg} / \mathrm{m}^{2} ; n=4$ individuals) were incubated with vehicle or AICAR ( $1 \mathrm{mmol} / \mathrm{l}$ ) for $6 \mathrm{~h}$. (a) Tissue macrophages were characterised as M1 $\left(\mathrm{CD} 11 \mathrm{c}^{+}\right), \mathrm{M} 1 / \mathrm{M} 2 \mathrm{~b}\left(\mathrm{CD} 86^{+}\right), \mathrm{M} 2 \mathrm{a}\left(\mathrm{CD} 206^{+}\right)$or M2a/M2c $\left(\mathrm{CD} 163^{+}\right)$ and (b) quantified. (c) $\mathrm{T}$ cells were characterised as $\mathrm{CD} 4^{+}$and $\mathrm{CD} 8^{+}$ and (d) quantified. (e) Levels of TNF- $\alpha$ in the supernatant fraction were determined by ELISA. Data are presented as mean \pm SEM. $* p<0.05$, $* * * p<0.001$, Student's $t$ tests 

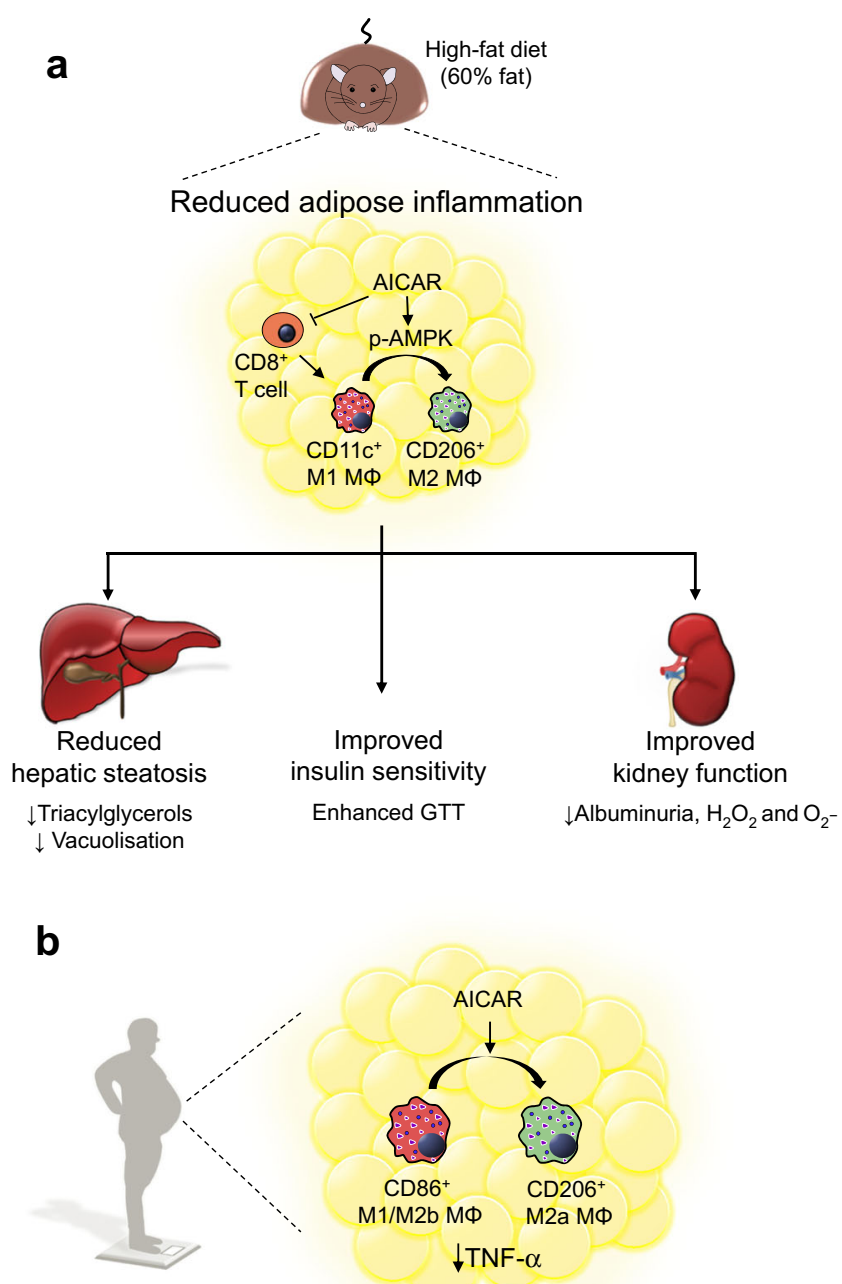

Fig. 7 Schematic illustration of proposed AICAR-mediated effects in obesity. (a) In obese mice, AICAR treatment attenuates HFD-induced adipose inflammation, promoting an M1-to-M2 macrophage phenotype switch by reducing $\mathrm{CD} 8^{+} \mathrm{T}$ cell infiltration, while increasing $\mathrm{p}$-AMPK levels. This results in reduced liver and kidney disease and enhanced glucose tolerance. All of these effects are independent of adiponectin. (b) AICAR mediates a similar M1-to-M2 macrophage phenotype switch in adipose explants isolated from obese individuals undergoing bariatric surgery. $\mathrm{M} \Phi$, macrophage

macrophages infiltrating the obese WAT produce proinflammatory mediators (TNF- $\alpha$, IL- $1 \beta$, IL-6), which are associated with the development of insulin resistance and the subsequent release of NEFA, leading to systemic lipotoxicity, with effects on the liver and kidney [26-28, 35]. AICAR treatment promoted an M1-to-M2 macrophage phenotype switch, reducing the percentage of HFD-induced CD11 $\mathrm{c}^{+}$ M1 macrophages, while restoring the $\mathrm{CD} 206^{+} \mathrm{M} 2$ macrophage population. Furthermore, AICAR increased WAT AMPK activity, which has been shown to promote an IL-10producing M2 macrophage phenotype [36-38]. In cultured macrophages, AICAR also promoted an M1-to-M2 phenotype switch and increased AMPK activation, suggesting that the drug may directly manipulate macrophage cell signalling and phenotypic responses. Additionally, AICAR treatment reduced HFD-induced $\mathrm{CD} 8^{+} \mathrm{T}$ cell infiltration, which may have contributed to the attenuated inflammation since $\mathrm{CD} 8^{+}$ T cells facilitate WAT accumulation of inflammatory CD $11 \mathrm{c}^{+}$ M1 macrophages [39].

Hepatic steatosis is associated with obesity and diabetes and enhances susceptibility to liver disease [40, 41]. AICAR inhibited hepatic steatosis, reducing HFD-induced hepatic triacylglycerol accumulation in both wild-type and Adipo ${ }^{-/-}$ mice. This is in agreement with earlier studies demonstrating that AICAR reduces diet-induced hepatic triacylglycerol content in rats [9] and TNF- $\alpha$-induced intracellular triacylglycerol accumulation in human hepatic HepG2 cell lines [11]. AICAR also attenuated HFD-induced hepatic cholesterol accumulation in Adipo ${ }^{-/-}$mice, an interesting finding since AICAR-induced activation of AMPK inhibits the hepatic thyroid stimulating hormone (TSH)/sterol regulatory element-binding protein-2 (SREBP-2)/3-hydroxy-3-methylglutaryl-CoA reductase (HMGCR) pathway necessary for cholesterol biosynthesis [10].

Obesity is an independent risk factor for kidney disease and $25-40 \%$ of diabetic individuals develop nephropathy, which is the primary cause of end-stage renal failure $[1,2,18]$. In this study, we demonstrate that AICAR treatment attenuates cardinal features of HFD-induced kidney disease, namely microalbuminuria, production of reactive oxygen species and renal inflammation. AICAR did not affect the total number of renal macrophages in the mouse model used, but rather shifted their phenotype towards resolution by reducing the percentage of $\mathrm{CD} 11 \mathrm{c}^{+} \mathrm{M} 1$ macrophages. Collectively, this supports previous work from our group and others, demonstrating that AICAR attenuates both HFD-induced $[12,13]$ and diabetes-induced $[14,15]$ kidney disease. Importantly, in this study, we now also demonstrate that AICAR-mediated protection against kidney disease is independent of adiponectin. This is critical to the clinical application of AICAR as a potential therapeutic agent targeting kidney disease. Indeed, it has been debated whether the use of AICAR could be compromised in patients with diabetic nephropathy because research indicates that the drug may increase adiponectin [13]. Although adiponectin is generally described as a protective adipokine [16] and thought to protect podocyte function in early onset kidney disease [18, 42-44], the role of adiponectin in the later stages of human renal failure is unclear [18] with some studies suggesting that it is harmful [19, 20]. Thus, our finding that AICAR attenuated HFD-induced kidney disease in an adiponectin-independent manner may indicate that the drug is a more suitable therapeutic agent for patients with advanced nephropathy.

Importantly, Adipoq ${ }^{-1-}$ mice presented with increased inflammation, liver vacuolisation and kidney injury compared with wild-type mice, probably because of the lack of the 
protective hormone adiponectin. Despite increased injury in the Adipo $^{-/-}$strain, AICAR maintained protection against WAT inflammation and liver and kidney injury. Thus, we conclude that this AICAR-mediated protection is independent of adiponectin. However, although AICAR maintained renoprotective effects in Adipo $q^{-1-}$ mice, it did not reduce the increased levels of renal $\mathrm{CD} 11 \mathrm{c}^{+} \mathrm{M} 1$ macrophages observed in these mice. Thus, these data indicate that AICAR-mediated renal protection is not mediated via reduced renal inflammation per se, but rather we hypothesise that the protection derives from the reduction of adipose inflammation, as illustrated in Fig. 7.

To translate our rodent data to human pathophysiology, we investigated if AICAR could reduce WAT inflammation in humans. AICAR promoted an M1-to-M2 macrophage phenotype shift in human WAT explants obtained from obese individuals. However, we observed important differences in the specific macrophage phenotypes affected in mice vs humans. AICAR did not affect $\mathrm{M} 1 \mathrm{CD} 11 \mathrm{c}^{+}$macrophage expression in human WAT, although M1/M2b CD86 $6^{+}$macrophage expression was reduced. This may be because AICAR did not affect the number of human $\mathrm{CD} 8^{+} \mathrm{T}$ cells, which drive $\mathrm{CD}_{11 \mathrm{c}^{+}}$macrophage infiltration [39]. Importantly, the ex vivo culture of human tissue with AICAR was limited to $6 \mathrm{~h}$; thus it is possible that continuous therapeutic administration of the drug to patients may promote more substantial modulation of $\mathrm{T}$ cell and macrophage phenotypes. AICAR acted in a proresolving manner by increasing the anti-inflammatory CD206 ${ }^{+}$macrophage population in human WAT. Since $\mathrm{CD}_{163^{+}}$macrophage expression remained unaffected, AICAR may specifically promote the M2a phenotype. Finally, AICAR attenuated the level of TNF- $\alpha$ in human WAT, which is a key functional response in promoting metabolic health.

Collectively, these data support the use of AICAR to promote metabolic health and to protect against obesityinduced pathophysiology, such as liver steatosis and kidney disease. WAT inflammation is a common denominator of obesity-related pathologies, causing systemic lipotoxicity, insulin resistance and organ dysfunction. Thus, it is noteworthy that AICAR reduces WAT inflammation in both mice and humans. Importantly, AICAR protects against disease in an adiponectin-independent manner, which may make AICAR a suitable therapy for individuals with nephropathy.

Acknowledgements Scientific input and technical support is acknowledged from: S.-E. Thörn and the nurses at SU-East Hospital, M. Engström, N. Björnfot, V. Sorhegui and A. Ferraro Werling (Department of Gastrosurgical Research and Education, Sahlgrenska Academy, University of Gothenburg, Sweden); I. Bergström (Department of Medical and Health Science [IMH], Linköping University, Sweden); P. Akeus and P. Sundström (Department of Biomedicine, University of Gothenburg, Sweden); D. Sirypangno
(Flow Core, VA, USA); L. Slater (Centre for Renal Translational Medicine [CRTM], UC San Diego [UCSD], CA, USA); L. Patterson and A. Andreasson (VA vivarium, UCSD, CA, USA); and S. Chavez, M. Clark and L. Gauthier (students at CRTM, UCSD, CA, USA). We acknowledge valuable editorial assistance from R. Perkins at the Wallenberg Laboratory, University of Gothenburg, Sweden. Some of the data were presented as an abstract at the Experimental Biology meeting in 2016, titled 'Therapeutic potential of AICAR in attenuating obesity-induced metabolic, liver and kidney disease'.

Funding There are no competing financial interests by the authors. EB is supported by the Swedish Research Council, (no. 2016/82), the Swedish Society for Medical Research (no. S150086), Åke Wiberg's Foundation (no. M15-0058), Konrad and Helfrid Johansson's Foundation (no. Borgeson2016) and the Wallenberg Centre for Molecular and Translational Medicine in Gothenburg, Sweden. EB was previously supported by a Marie Curie International Outgoing Fellowship (IOF-GA-2011-301803). LF and VW are supported by grants from the Western Region of Sweden (Strategic ALF grants, no. ALFGBG-442371) and the Erik \& Lily Philipson memorial foundation (no. 121818). CG is supported by Science Foundation Ireland (15/US/B3130 and 15/IA/152) and the National Institute of Diabetes and Digestive and Kidney Diseases (NIDDK) Diabetic Complications Consortium (DiaComp, www.diacomp. org; grant DK076169). KS is supported by VA Merit Grant (5I01 BX000277).

Data availability Data supporting the results reported in the article can be found in the Sharma laboratory (kumarsharma@ucsd.edu).

Duality of interest The authors declare that there is no duality of interest associated with this manuscript.

Contribution statement EB, KS, and CG conceived and designed the study; EB, JLR, GHS, MD, VW, MRE, PB and CB contributed to data acquisition; EB, VW, LF and MQ-J analysed the data; all authors interpreted the data, drafted the article, revised it critically for important intellectual content and approved the final version to be published. KS is the guarantor of this work and, as such, had full access to all the data in the study and takes responsibility for the integrity of the data and the accuracy of the data analysis.

Open Access This article is distributed under the terms of the Creative Commons Attribution 4.0 International License (http:// creativecommons.org/licenses/by/4.0/), which permits unrestricted use, distribution, and reproduction in any medium, provided you give appropriate credit to the original author(s) and the source, provide a link to the Creative Commons license, and indicate if changes were made.

\section{References}

1. Borgeson E, Sharma K (2013) Obesity, immunomodulation and chronic kidney disease. Curr Opin Pharmacol 13:618-624

2. Mathew AV, Okada S, Sharma K (2011) Obesity related kidney disease. Curr Diabetes Rev 7:41-49

3. NCD Risk Factor Collaboration (2016) Trends in adult body-mass index in 200 countries from 1975 to 2014: a pooled analysis of 1698 population-based measurement studies with 19.2 million participants. Lancet 387:1377-1396

4. Viollet B, Guigas B, Leclerc J et al (2009) AMP-activated protein kinase in the regulation of hepatic energy metabolism: from physiology to therapeutic perspectives. Acta Physiol 196:81-98 
5. Misra P, Chakrabarti R (2007) The role of AMP kinase in diabetes. Indian J Med Res 125:389-398

6. Aschenbach WG, Hirshman MF, Fujii N, Sakamoto K, Howlett KF, Goodyear LJ (2002) Effect of AICAR treatment on glycogen ?metabolism in skeletal muscle. Diabetes 51:567-573

7. Gaidhu MP, Frontini A, Hung S, Pistor K, Cinti S, Ceddia RB (2011) Chronic AMP-kinase activation with AICAR reduces adiposity by remodeling adipocyte metabolism and increasing leptin sensitivity. J Lipid Res 52:1702-1711

8. Narkar VA, Downes M, Yu RT et al (2008) AMPK and PPAR $\delta$ agonists are exercise mimetics. Cell 134:405-415

9. Henriksen BS, Curtis ME, Fillmore N, Cardon BR, Thomson DM, Hancock CR (2013) The effects of chronic AMPK activation on hepatic triglyceride accumulation and glycerol 3-phosphate acyltransferase activity with high fat feeding. Diabetol Metab Syndr 5: 29

10. Liu S, Jing F, Yu C, Gao L, Qin Y, Zhao J (2015) AICAR-induced activation of AMPK inhibits TSH/SREBP-2/HMGCR pathway in liver. PLoS One 10:e124951

11. Lv Q, Zhen Q, Liu L et al (2015) AMP-kinase pathway is involved in tumor necrosis factor alpha-induced lipid accumulation in human hepatoma cells. Life Sci 131:23-29

12. Decleves AE, Zolkipli Z, Satriano J et al (2014) Regulation of lipid accumulation by AMP-activated kinase in high fat diet-induced kidney injury. Kidney Int 85:611-623

13. Decleves AE, Mathew AV, Cunard R, Sharma K (2011) AMPK mediates the initiation of kidney disease induced by a high-fat diet. J Am Soc Nephrol 22:1846-1855

14. Dugan LL, You YH, Ali SS et al (2013) AMPK dysregulation promotes diabetes-related reduction of superoxide and mitochondrial function. J Clin Invest 123:4888-4899

15. Lee MJ, Feliers D, Mariappan MM et al (2007) A role for AMPactivated protein kinase in diabetes-induced renal hypertrophy. Am J Physiol Ren Physiol 292:F617-F627

16. Kadowaki T, Yamauchi T (2005) Adiponectin and adiponectin receptors. Endocr Rev 26:439-451

17. Ortega Moreno L, Lamacchia O, Salvemini L et al (2016) The paradoxical association of adiponectin with mortality rate in patients with type 2 diabetes: evidence of synergism with kidney function. Atherosclerosis 245:222-227

18. Sweiss N, Sharma K (2014) Adiponectin effects on the kidney. Best Pract Res Clin Endocrinol Metab 28:71-79

19. Saraheimo M, Forsblom C, Fagerudd J et al (2005) Serum adiponectin is increased in type 1 diabetic patients with nephropathy. Diabetes Care 28:1410-1414

20. Saraheimo M, Forsblom C, Thorn L et al (2008) Serum adiponectin and progression of diabetic nephropathy in patients with type 1 diabetes. Diabetes Care 31:1165-1169

21. Moller KF, Dieterman C, Herich L, Klaassen IA, Kemper MJ, Muller-Wiefel DE (2012) High serum adiponectin concentration in children with chronic kidney disease. Pediatr Nephrol 27:243249

22. Kim HY, Bae EH, Ma SK et al (2016) Association of serum adiponectin level with albuminuria in chronic kidney disease patients. Clin Exp Nephrol 20:443-449

23. Nanayakkara PW, Le Poole CY, Fouque D et al (2009) Plasma adiponectin concentration has an inverse and a non linear association with estimated glomerular filtration rate in patients with K/DOQI 3-5 chronic kidney disease. Clin Nephrol 72:21-30
24. Menon V, Li L, Wang X et al (2006) Adiponectin and mortality in patients with chronic kidney disease. J Am Soc Nephrol 17:2599 2606

25. Börgeson E (2016) The role of lipoxins in cardiometabolic physiology and disease. Cardiovasc Endocrinol 5:4-13

26. McNelis JC, Olefsky JM (2014) Macrophages, immunity, and metabolic disease. Immunity 41:36-48

27. Olefsky JM, Glass CK (2010) Macrophages, inflammation, and insulin resistance. Annu Rev Physiol 72:219-246

28. Weisberg SP, McCann D, Desai M, Rosenbaum M, Leibel RL, Ferrante AW Jr (2003) Obesity is associated with macrophage accumulation in adipose tissue. J Clin Invest 112:1796-1808

29. Borgeson E, Johnson AM, Lee YS et al (2015) Lipoxin A attenuates obesity-induced adipose inflammation and associated liver and kidney disease. Cell Metab 22:125-137

30. Andrikopoulos S, Blair AR, Deluca N, Fam BC, Proietto J (2008) Evaluating the glucose tolerance test in mice. Am J Physiol Endocrinol Metab 295:E1323-E1332

31. Borgeson E, McGillicuddy FC, Harford KA et al (2012) Lipoxin A4 attenuates adipose inflammation. FASEB J 26:4287-4294

32. Teteris SA, Hochheiser K, Kurts C (2012) Isolation of functional dendritic cells from murine kidneys for immunological characterization. Nephrology 17:364-371

33. Martinez FO, Sica A, Mantovani A, Locati M (2008) Macrophage activation and polarization. Front Biosci 13:453-461

34. Lumeng CN, Bodzin JL, Saltiel AR (2007) Obesity induces a phenotypic switch in adipose tissue macrophage polarization. J Clin Invest 117:175-184

35. Gonzalez-Periz A, Claria J (2010) Resolution of adipose tissue inflammation. Sci World J 10:832-856

36. Zhu YP, Brown JR, Sag D, Zhang L, Suttles J (2015) Adenosine 5'monophosphate-activated protein kinase regulates IL-10-mediated anti-inflammatory signaling pathways in macrophages. J Immunol 194:584-594

37. Koscso B, Csoka B, Kokai E et al (2013) Adenosine augments IL10-induced STAT3 signaling in M2c macrophages. J Leukoc Biol 94:1309-1315

38. Sag D, Carling D, Stout RD, Suttles J (2008) Adenosine 5'monophosphate-activated protein kinase promotes macrophage polarization to an anti-inflammatory functional phenotype. J Immunol 181:8633-8641

39. Nishimura S, Manabe I, Nagasaki M et al (2009) CD8+ effector T cells contribute to macrophage recruitment and adipose tissue inflammation in obesity. Nat Med 15:914-920

40. Spite M, Claria J, Serhan CN (2014) Resolvins, specialized proresolving lipid mediators, and their potential roles in metabolic diseases. Cell Metab 19:21-36

41. Fabbrini E, Magkos F (2015) Hepatic steatosis as a marker of metabolic dysfunction. Nutrients 7:4995-5019

42. Ohashi K, Iwatani H, Kihara S et al (2007) Exacerbation of albuminuria and renal fibrosis in subtotal renal ablation model of adiponectin-knockout mice. Arterioscler Thromb Vasc Biol 27: 1910-1917

43. Rutkowski JM, Wang ZV, Park AS et al (2013) Adiponectin promotes functional recovery after podocyte ablation. J Am Soc Nephrol 24:268-282

44. Sharma K, Ramachandrarao S, Qiu G et al (2008) Adiponectin regulates albuminuria and podocyte function in mice. $\mathrm{J}$ Clin Invest 118:1645-1656 